\title{
RETRACTED ARTICLE: Ecological stability of the shorebird stopover site in the Yalu River Estuary Wetlands, China
}

\author{
Lun Song • Guojun Yang • Liqiang Zhao • \\ Yanan $\mathbf{L u} \cdot \mathbf{N a n} \mathbf{L i} \cdot$ Nianbin Wang
}

Received: 6 August 2013/Accepted: 31 January 2014/Published online: 23 February 2014

(C) Springer Science+Business Media Dordrecht 2014

The Editors in Chief of Wetlands Ecology and Management received a complaint from an alert reader to the effect that the paper 'Ecological stability of the shorebird stopover site in the Yalu River estuary wetlands, China' by Lun Song, Guojun Yang, Liqiang Zhao, Yanan Lu, Nan Li and Nianbin Wang, published as Online First article in Wetlands Ecology and Management is a plagiarized version of an earlier articles published in: 1) 'A study on bioecology of the stopover site of waders within China's Yalu River estuary wetlands by Song L., Yang G.J., Li A. and Wang N.B. (Acta Ecologica Sinica, 2011, Vol. 31(24): 7500-7510) and 2) 'The stress response of biological communities in Chins's Yalu River Estuary and neighboring waters' (Acta Ecologica Sinica, 2013, Vol. 33 (9):2790-2802). The 'results' part of the Wetlands Ecology and Management paper contained exactly the same tables and figures of results as those of the articles published in 2011 and 2013. Accordingly, and as per the journal's policy on plagiarism, it has been decided to withdraw the article published by Lun Song, Guojun Yang, Liqiang Zhao, Yanan Lu, Nan Li and Nianbin Wang in Wetlands Ecology and Management. The Editorial Board takes a serious view of acts of plagiarism in the journal and is committed to take all necessary steps to prevent such acts.
L. Song $(\bowtie) \cdot$ N. Li $\cdot$ N. Wang

Key Laboratory of Marine Biological Resources and Ecology, Liaoning Province, Liaoning Ocean and Fisheries Science Research Institute, Dalian 116023 , China

e-mail: lun_song@163.com

G. Yang - L. Zhao - Y. Lu

College of Fisheries and Life science, Dalian Ocean University, Dalian 116023, China 